\title{
The Japanese Translation of the Life Engagement Test: Reliability and Construct Validity in a College Student Population and a Working Adult Population
}

Katsunori Sumi

Nagare College, Nagoya Institute of Technology, Gokiso-cho, Showa-ku, Nagoya, 466-8555, Japan

\section{Abstract}

The Life Engagement Test is a self-report measure of purpose in life. The purpose of the present study was to examine the reliability and construct validity of a Japanese translation of the Life Engagement Test (LET-J) in two samples of 409 Japanese college students (166 women, 263 men; mean age $=21.07$ years, $S D=1.14$ ) and 439 adult workers ( 232 women, 207 men; mean age $=39.79$ years, $S D=11.23$ ). The LET-J was found to have acceptable internal consistency reliability in both samples ( $\alpha \mathrm{s}=.82$ to .86 ) and testretest reliability in the student sample $(r=.76)$. Exploratory factor analysis and subsequent confirmatory factor analysis supported the expected one-factor structure of the LET-J in both samples. The expected correlations with scores on the hedonic and eudaimonic well-being measures were generally found common to both samples, supporting the discriminant and convergent validity of the LET-J. The LET-J was shown to be a useful measure that will certainly contribute to research on purpose in life in the Japanese population.

\section{Introduction}

Purpose in life has received considerable attention in preceding literature, perhaps with the growing focus on positive psychology [1]. In general, the construct of purpose in life refers to a global belief in one's life purpose [2], and has been identified as one of the fundamental dimensions of eudaimonic well-being [3-5]. Previous findings have shown that purpose in life has a positive effect on mental and physical health outcomes [6], including a diminished mortality risk for younger as well as older adults $[7,8]$. Purpose in life may be an important factor to promote and improve our well-being, and therefore a measure to properly access purpose in life is significant.

In most previous studies, purpose in life has been assessed by several measures [7, 9], including the Purpose in Life Test [10] and the purpose in life sub-scale of the Psychological Well-being Scale [11], which have been used often [9]. However, some problems have been pointed out in these existing measures. First, the measures often include items that assess constructs other than purpose in life, for example, meaning in life and satisfaction with life [12]. Although it has been suggested that there are close relationships among these constructs $[11,13,14]$, some authors treat purpose in life as an independent construct from meaning in life and satisfaction with life $[15,16]$. It has been argued that such confounding of the construct which the existing measures assess, is inappropriate [12]. Second, the existing measures also include both items about both a current purpose in life and items about the sense of having had purpose in one's life to date $[12,17]$. It has been suggested that this time insensitivity is inadequate for purpose in life measure, because it prevents assessing temporal changes of purpose in life [12].

A measure of purpose in life that eliminated the problems in the existing measures is the Life Engagement Test (LET) [12], a short measure that has been widely used by many researchers. The LET is designed to assess the extent to which a person engages in personally valued activities [12]. The psychometric properties of the LET have been assessed in eight samples, which included different genders, ages, and ethnic groups [12]. A one-factor solution has been found through exploratory factor analysis for all the samples. The LET has shown acceptable internal consistency reliability in all samples and groups (Cronbach's alphas $=.72$ to .87 ), and moderate temporal stability over approximately four months in four samples ( $r s=.61$ to .76$)$. Convergent validity of the LET was supported through significant correlation with many psychosocial and health-relevant variables, such as self-esteem, hostile affect, the Big 5 personality traits, mental health, social support, and life satisfaction. In addition, discriminant validity was supported by the finding that well-being scores were more closely related to the LET scores than scores on the purpose in life subscale of the Psychological Well-being Scale [11].

A Japanese version of the LET, which is a validated and useful measure, should promote research on purpose in life in Japan. The purpose of this study was to translate the LET into Japanese and to examine the reliability and construct validity of the translated version (LET-J) among college students and adult workers. Testretest reliability was examined over a four-week period in the college students.

Construct validity of the LET-J was examined by correlating scores on the scale with well-being measures. Although it has been supported that purpose in life is closely related to well-being, purpose in life is not a construct that overlaps with all the components of well-being [12]. For example, hedonic well-being, which consists of cognitive and affective components [3,18], should be distinguished from purpose in life. Scheier et al. [12] found that the LET scores were moderately correlated with scores for life satisfaction $(r s=.34$ to .58 in seven distinct samples) as the cognitive component, and positive

"Corresponding Author: Prof. Katsunori Sumi, Nagare College, Nagoya Institute of Technology, Gokiso-cho, Showa-ku, Nagoya, 466-8555, Japan; E-mail: sumi@nitech.ac.jp

Citation: Sumi K (2018) The Japanese Translation of the Life Engagement Test: Reliability and Construct Validity in a College Student Population and a Working Adult Population. Int J Psychol Behav Anal 4: 136. doi: https://doi. org/10.15344/2018/2455-3867/136

Copyright: (C) 2017 Sumi. This is an open-access article distributed under the terms of the Creative Commons Attribution License, which permits unrestricted use, distribution, and reproduction in any medium, provided the original author and source are credited. 
affect $(r=.59)$ and negative affect $(r=-.28)$ as the affective component. From these results, Scheier et al. [12] concluded that purpose in life as measured by the LET should be distinguished from hedonic wellbeing. Therefore, moderate correlations between scores on the LET-J and hedonic well-being measure would support the discriminant validity of the LET-J. On the other hand, because purpose in life has been identified as one of the major dimension of eudaimonic wellbeing [3-5], a high correlation with scores on eudaimonic well-being measure would indicate convergent validity of the LET-J.

\section{Method}

\section{Participants}

The participants in this study were divided into two groups: college students (Sample 1) and adult workers (Sample 2). Sample 1 was composed of 399 Japanese students from two colleges in urban areas in Japan and consisted of 182 women and 217 men with a mean age of 21.07 ( $S D=1.14$, range 19 to 33 years). Sample 2 was composed of 439 full-time working adults and consisted of 207 women and 232 men with a mean age of $39.79(S D=11.23$, range 20 to 66 years). They had various occupations, which mainly included clerical (33.9\%), factory (32.1\%), and sales person work (12.7\%).

\section{Measures}

Life Engagement Test: The original LET, which appeared in English, was translated into Japanese using a translation and back-translation procedure [19]. One bilingual researcher translated the items of the LET into Japanese, and the other researcher translated the items from Japanese to English. Then, consistency between the translation and the back translation was checked by two researchers. This process was followed repeatedly until an acceptable degree of consistency was achieved. Because the items of the original LET are written plainly and express familiar experiences to Japanese people, cultural relevancy and item difficulty should have little influence on the translation process. After that, four graduate students confirmed that there was no problem with understanding the translated items.

The LET comprises six items, which were rated on a five-point scale ranging from 1 (strongly disagree) to 5 (strongly agree). Half of the items are positively worded (e.g., "I value my activities a lot"), and the remaining half is negatively worded (e.g., "There is not enough purpose in my life"). After reverse scoring negatively worded items, all the scores are summed. The range of possible scores is 6 to 30 , with higher scores indicating greater purpose in life.

Well-being measures: To assess the construct validity of the LET, hedonic and eudaimonic well-being measures were included in the present study. The cognitive and affective components of hedonic well-being were assessed using measures of life satisfaction and positive and negative affect, respectively. Eudaimonic well-being was assessed using a measure of psychosocial flourishing.

Satisfaction with Life Scale: The Japanese version of the five-item Satisfaction with Life Scale $[20,21]$ was used to measure life satisfaction as the cognitive component of hedonic well-being. The Japanese version uses a 5-point response format ranging from 1 (strongly disagree) to 5 (strongly agree). Acceptable internal consistency ( $\alpha=$ .84 ), factorial validity (with one factor), and negative correlation with depression $(r=-.30)$ have been reported for the Japanese version [21].
Scale of Positive and Negative Experience: Positive and negative affect was assessed using the Japanese version of the 12-item Scale of Positive and Negative Experience $[4,22,23]$ as the affective components of hedonic well-being. This scale comprises two subscales: a six-item positive affect scale and six-item negative affect scale. These items use a five-point response format ranging from 1 (very rarely or never) to 5 (very often or always). The subscales have good internal consistency $(\alpha s=.86$ to .93$)$ and test-retest reliability over one month $(r s=.60$ and .57) $[22,23]$. Exploratory and confirmatory factor analysis showed a two factor structure for the Japanese version [22]. Acceptable convergent validity was supported by correlations with scores on several well-being measures [22].

Flourishing Scale: Psychosocial flourishing as eudaimonic well-being was measured using the Japanese version of the eight-item Flourishing Scale $[4,22,23]$. This scale has a seven-point response format ranging from 1 (strongly disagree) to 7 (strongly agree). The sale has good internal consistency $(\alpha=.94$ to .95$)$ and test-retest reliability over one month $(r=.87)$ [22,23]. Exploratory and confirmatory factor analysis indicated the viability of a one-factor model for the Japanese version [22]. Acceptable convergent validity was provided through correlations with scores on several well-being measures [22].

\section{Procedure of questionnaire administration}

All the participants in Sample 1 took part in two questionnaire sessions separated by a 4 -week interval (Time 1 and Time 2). They completed a Japanese translation of the LET at both Time 1 and Time 2 and well-being measures only at Time 1 . On the other hand, the participants in Sample 2 completed the Japanese translation and well-being measures only once. The participants of Sample 1 and 2 participated in this study voluntarily after informed consent was obtained. Ethical clearance for the study was obtained from the relevant ethical committee.

\section{Data analysis}

First, to assess the internal consistency reliability of the LET-J, corrected item-total correlations and Cronbach's alphas were calculated. Second, Test-retest reliability was examined using the data at Time 1 and Time 2 from Sample 1. Third, to examine the one-factor structure of the LET-J, exploratory and confirmatory factor analysis was conducted. Each sample was randomly divided into two approximately equal-sized subsamples. Exploratory factor analysis using principal axis factoring was first performed to explore the underlying factor structure of the LET-J using the data from one sample, and then confirmatory factor analysis was used to test the factor structure on the data from the other. Finally, discriminant and convergent validity was examined by assessing correlations between scores on the LET-J and the hedonic and eudaimonic well-being measures. These correlation coefficients were calculated from the data of Sample 1 at Time 1 and Sample 2. Before all the analyses, the three reversed items of the LET-J (i.e., items 1,3, and 5) were reverse coded.

\section{Results}

\section{Internal consistency and temporal stability}

The means, standard deviations, range of scores, corrected itemtotal correlations, and Cronbach's alphas for the LET-J in each sample are reported. The corrected item-total correlations were moderate to high $(r=.52$ to .72$)$. Cronbach's alphas were above $.80(.82$ to .86$)$ and 
Citation: Sumi K (2018) The Japanese Translation of the Life Engagement Test: Reliability and Construct Validity in a College Student Population and a Working Adult Population. Int J Psychol Behav Anal 4: 136. doi: https://doi.org/10.15344/2018/2455-3867/136

Page 3 of 5

about the same values across the samples. The correlation between LET-J scores at Time 1 and Time 2 in Sample 1 was very high $(r=.76$, $95 \%$ confidence interval $=.72, .80$ ).

\begin{tabular}{|l|l|l|l|l|l|l|}
\hline \multicolumn{2}{|l|}{} & M & SD & $\begin{array}{l}\text { Range of } \\
\text { Scores }\end{array}$ & CITC & Cronbach's $\alpha$ \\
\hline Sample 1 & Time 1 & 20.75 & 4.29 & $8-30$ & $.52-.69$ & .82 \\
\hline & Time 2 & 20.98 & 4.38 & $6-30$ & $.57-.72$ & .86 \\
\hline Sample 2 & & 21.42 & 4.10 & $6-30$ & $.52-.70$ & .83 \\
\hline
\end{tabular}

Table 1: Means, Standard Deviations, Range of Scores, and Cronbach's a of LET-J.

Note. CITC $=$ corrected item-total correlations

\section{Factor structure}

Participants in Sample 1 and Sample 2 were randomly divided into Sample 1a $(n=200)$ and Sample $1 \mathrm{~b}(n=199)$, and Sample 2a $(n=$ $220)$ and Sample $2 \mathrm{~b}(n=219)$, respectively. There were no significant differences between Sample 1a and Sample $1 \mathrm{~b}$ with regard to sex, $\chi^{2}(1$, $N=399)=.05$, and age, $t(397)=.13$. Like Sample 1, there was no significant difference between Sample 2a and Sample 2b with regard to sex, $\chi^{2}(1, \mathrm{~N}=439)=.02$, and age, $t(437)=.02$. In addition, no significant differences in mean scores for all the scales were found between Sample 1a and Sample 1b and between Sample 2a and Sample 2b.

The exploratory factor analysis was performed on the data from Sample 1a at Time 1 and Time 2 and the data from Sample 2a. The Kaiser-Meyer-Olkin measures of sampling adequacy were .85, 84, and .78 , and Bartlett's tests of sphericity were $408.72,614.30$, and 508.56 ( $p$ s $<.01)$, respectively. These results indicated that the data collected were appropriate for the factor analysis. The exploratory factor analysis with each sample extracted only one factor with eigenvalues greater than 1.0, accounting for $58.07 \%, 62.35 \%$, and $53.79 \%$ of the total variance in Sample 1a at Time 1 and Time 2 and Sample 2a, respectively. As shown in Table 2, the factor loadings of the items were all greater than .55

To assess the adequacy of the one-factor model, confirmatory factor analysis was conducted on the data from Sample $1 \mathrm{~b}$ at Time 1 and Time 2 and those from Sample 2b. As shown in Table 3, goodness of fit indices indicated an acceptable fit of the one-factor model to the data for the groups. Table 2 also includes standardized factor loadings for each group. The factor loadings were all significant $(p s<.01)$, and larger than .50 .

\section{Correlations with Scores on Hedonic and Eudaimonic Well-being Measures}

Table 4 shows the Pearson correlations between scores on the LET-J and well-being measures. In general, the expected correlations were found between the scores on these measures. In addition, these correlations were similar between the two samples. In both samples, LET-J scores were moderately positively correlated with scores on the Satisfaction with Life Scale and Positive Affect scale ( $r s=.37$ to .54 ). Although the correlations between scores on the LET-J and Negative Affect scale were negative and significant ( $\mathrm{rs}=-.19$ and -.29), the magnitude of these correlations was somewhat lower than those between the LET-J scores and scores for life satisfaction and positive affect measures. These correlations with the hedonic well-being measures support the discriminant validity of the LET-J. In contrast, scores on the Flourishing Scale were very highly correlated with the LET-J scores ( $r$ s $=.71$ and .70$)$, supporting the convergent validity of the LET-J.

\begin{tabular}{|l|c|c|c|c|c|c|}
\hline \multirow{2}{*}{$\begin{array}{l}\text { Item } \\
\text { No. }\end{array}$} & \multicolumn{3}{|c|}{ Exploratory Factor Analysis } & \multicolumn{3}{c|}{ Confirmatory Factor Analysis } \\
\cline { 2 - 5 } & \multicolumn{2}{|c|}{ Sample 1a } & Sample 2a & \multicolumn{2}{c|}{ Sample 1b } & Sample 2b \\
\cline { 2 - 5 } & Time 1 & Time 2 & & Time 1 & Time 2 & \\
\hline 1 & .60 & .57 & .64 & .53 & .70 & .54 \\
\hline 2 & .62 & .73 & .55 & .66 & .69 & .54 \\
\hline 3 & .71 & .75 & .69 & .75 & .74 & .68 \\
\hline 4 & .75 & .81 & .63 & .77 & .76 & .63 \\
\hline 5 & .79 & .79 & .78 & .68 & .70 & .84 \\
\hline 6 & .76 & .79 & .72 & .73 & .69 & .76 \\
\hline
\end{tabular}

Table 2: Factor Loadings for Exploratory and Confirmatory Factor Analysis.

aFor confirmatory factor analyses, standardized factor loadings are hown. All the factor loadings are significant at the .01 alpha level.

\begin{tabular}{|l|l|l|l|l|l|l|l|}
\hline & & $\chi^{2 \mathrm{a}}$ & GFI & AGFI & RMSEA & SRMR & CFI \\
\hline \multirow{2}{*}{ Sample 1b } & Time 1 & $16.26^{*}$ & .98 & .93 & .08 & .03 & .98 \\
\cline { 2 - 8 } & Time 2 & $17.41^{*}$ & .97 & .92 & .09 & .03 & .98 \\
\hline Sample 2b & & $19.33^{* *}$ & .98 & .91 & .10 & .04 & .97 \\
\hline
\end{tabular}

Table 3: Goodness of Fit Indices for Sample $1 \mathrm{~b}$ and $2 \mathrm{~b}$.

Note. GFI = goodness of fit index; AGFI = adjusted goodness of fit index; RMSEA = root mean square error of approximation; SRMR = standardized root mean square residual; $\mathrm{CFI}=$ comparative fit index.

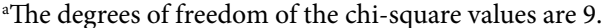
${ }^{*} p<.05,{ }^{* *} p<.01$.

\begin{tabular}{|l|l|l|l|l|l|l|}
\hline & & $r$ & $95 \%$ CI & $M$ & SD & Cronbach's $\alpha$ \\
\hline \multirow{4}{*}{ Sample 1 } & Satisfaction with Life Scale & .37 & {$[.28, .45]$} & 13.28 & 3.45 & .73 \\
\cline { 2 - 7 } & Positive Affect scale & .48 & {$[.40, .55]$} & 12.01 & 4.51 & .92 \\
\cline { 2 - 7 } & Negative Affect scale & -.19 & {$[-.28,-.09]$} & 17.22 & 4.46 & .80 \\
\cline { 2 - 7 } & Flourishing Scale & .71 & {$[.66, .75]$} & 36.30 & 7.59 & .81 \\
\hline \multirow{4}{*}{ Sample 2 } & Satisfaction with Life Scale & .45 & {$[.37, .52]$} & 14.20 & 3.56 & .82 \\
\cline { 2 - 7 } & Positive Affect scale & .54 & {$[.47, .61]$} & 21.89 & 4.58 & .92 \\
\cline { 2 - 7 } & Negative Affect scale & -.29 & {$[-.37,-.20]$} & 16.77 & 4.83 & .87 \\
\cline { 2 - 7 } & Flourishing Scale & .70 & {$[.64, .74]$} & 37.08 & 6.71 & .80 \\
\hline
\end{tabular}

Table 4: Pearson Correlations between Scores on LET-J and Well-Being Measures.

Note. $\mathrm{r} 1=$ Pearson correlations with LET scores at Time 1; $\mathrm{r} 2=$ Pearson correlations with LET scores at Time 2; 95\% CI $=95 \%$ confidence interval.

${ }^{a}$ All significance probabilities are less than .01 . 
Citation: Sumi K (2018) The Japanese Translation of the Life Engagement Test: Reliability and Construct Validity in a College Student Population and a Working Adult Population. Int J Psychol Behav Anal 4: 136. doi: https://doi.org/10.15344/2018/2455-3867/136

Page 4 of 5

This study was conducted to translate the original English version of the LET into Japanese, and preliminarily assess its reliability and construct validity among two distinct Japanese populations: college students and adult workers. The results of the analysis, the analyses indicate that the reliability of the LET-J is acceptable in general, like the results of Scheier et al. [12]. The internal consistency reliability of the LET-J was considered good with Cronbach's alphas above .80 [24] for both samples. A very high correlation was found between the LET-J scores obtained four weeks apart for the college student sample. For the original LET, Scheier et al. [12] found high to very high correlations between the scores obtained approximately four months apart for four samples. In light of these findings for the original LET, the result for the LET-J in the present study probably indicates acceptable temporal stability.

The construct validity of the LET-J was supported by the expected factor structure and correlations with scores on the well-being measures. The exploratory and confirmatory factor analysis with both the college student sample and adult worker sample revealed a onefactor structure that was found in the exploratory factor analysis of the original LET [12]. These findings support the factorial validity of the LET-J across the two samples.

Moreover, as expected, the LET-J scores were moderately correlated with scores for life satisfaction and positive affect as hedonic wellbeing. Although there were somewhat weaker correlations with scores on the negative affect scale, Scheier et al. [12] also found a weaker correlation between the original LET scores and scores on the negative affect scale. Purpose in life may be more weakly related to negative affect, in comparison with other components of hedonic well-being. As suggested by Scheier et al. [12], these correlations indicate that the LET-J and the hedonic well-being measures appear to assess related but distinct constructs, supporting the discriminant validity of the LET-J. On the other hand, there was a very high correlation between scores on the LET-J and the measure of eudaimonic well-being, which includes purpose in life as its major dimension. This result confirms the convergent validity of the LET-J. Additionally, the correlations of the LET-J were generally similar across the two different samples. Therefore, these results support that the LET-J has similar construct validity, and internal consistency reliability, in both college student and adult worker populations.

The findings of this study generally supported that the LET-J is a useful instrument for assessing purpose in life with acceptable reliability and construct validity. However, several limitations must be noted and will guide future research. First, the sample of this study was limited to college students and adult workers. Future studies should examine the psychometric properties of the LET-J in other populations such as the elderly, non-working people, or clinical samples. Second, test-retest reliability over a longer period should be examined in future to ascertain the temporal stability of the LET-J scores at various intervals. Third, although in this study, the construct validity of the LET-J was examined through correlations with wellbeing measures, association with other purpose in life measures and measures of various health outcomes, as in Scheier et al's work [12], should be also examined for further study of the construct validity. Finally, the validity of the LET-J, including predictive and concurrent validity, should be investigated further.

\section{Competing Interests}

The authors declare that they have no competing interests.

\section{Acknowledgements}

The kind permission to translate and reproduce the Life Engagement Test by M. F. Scheier is appreciated.

\section{Funding}

This research was supported by JSPS KAKENHI Grant Number 15K04121 (https://www.jsps.go.jp/english/e-grants/).

\section{References}

1. Steger MF, Frazier P, Oishi P, Kaler M (2006) The Meaning in Life Questionnaire: Assessing the presence of and search for meaning in life. $J$ Couns Psychol 53: 80-93.

2. Wang MC, Lightsey OR, Pietruszka T, Uruk AC, Wells AG (2007) Purpose in life and reasons for living as mediators of the relationship between stress, coping, and suicidal behavior. J Posit Psychol 2: 195-204.

3. Deci EL, Ryan RM (2008) Hedonia, eudaimonia, and well-being: An introduction. J Happiness Stud 9: 1-11.

4. Diener E, Wirtz D, Tov W, Kim-Prieto C, Choi D, et al. (2010) New wellbeing measures: Short scales to assess flourishing and positive and negative feelings. Soc Indic Res 97: 143-156.

5. Ryff CD (1989) Happiness is everything, or is it? Explorations on the meaning of psychological well-being. J Personal Soc Psychol 57: 10691081.

6. Roepke AM, Jayawickreme E, Riffle OM (2014) Meaning and health: A systematic review. Appl Res in Qual Life 9: 1055-1079.

7. Cohen R, Bavishi C, Rozanski A (2016) Purpose in life and its relationship to all-cause mortality and cardiovascular events: A meta-analysis. Psychosom Med 78: 122-133.

8. Hill PL, Turiano NA (2014) Purpose in life as a predictor of mortality across adulthood. Psychol Sci 25: 1482-1486.

9. Pinquart $M(2002)$ Creating and maintaining purpose in life in old age: $A$ meta-analysis. Ageing Int 27: 90-114.

10. Crumbaugh JC, Maholick LT (1964) An experimental study in existentialism: The psychometric approach to Frankl's concept of noogenic neurosis. $J$ Clin Psychol 20: 200-207.

11. Ryff CD, Keyes CLM (1995) The structure of psychological well-being revisited. J Personal Soc Psychol 69: 719-727.

12. Scheier MF, Wrosch C, Baum A, Cohen S, Martire LM, et al. (2006) The life engagement test: Assessing purpose in life. J Behav Med 29: 291-298.

13. Steger MF (2009) Meaning in life. In: Lopez SJ, Snyder CR (Eds) Oxford Handbook of Positive Psychology (2nd edition), Oxford University Press, UK, pp. 679-687.

14. Steger MF, Kashdan TB (2007) Stability and specificity of meaning in life and life satisfaction over one year. J Happiness Stud 8: 161-179.

15. Diener E, Fujita F, Tay L, Biswas-Diener R (2012) Purpose, mood, and pleasure in predicting satisfaction judgments. Soc Indic Res 105: 333-341.

16. Garrosa-Hernández E, Carmona-Cobo I, Ladstätter F, Blancoa LM, CooperThomas HD (2013) The relationships between family-work interaction, jobrelated exhaustion, detachment, and meaning in life A day-level study of emotional well-being. J Work Organ Psychol 29: 169-177.

17. Pearson EL, Windsor TD, Crisp DA, Butterworth P, Pilkington PD, et al (2013) Normative data and longitudinal invariance of the Life Engagement Test (LET) in a community sample of older adults. Qual Life Res 22: 327331.

18. Diener E, Oishi S, Lucas RE (2003) Personality, culture, and subjective well-being: Emotional and cognitive evaluations of life. Annu Rev Psychol 54: 403-425

19. Brislin RW (1970) Back-translation for cross-cultural research. J Cross Cult Psychol 1: 185-216.

20. Diener E, Emmons RA, Larsen RJ, Griffin S (1985) The satisfaction with life scale. J Pers Assess 49: 71-75.
Int J Psychol Behav Anal

ISSN: 2456-3501
IJPBA, an open access journal

Volume 4. 2018. 136 
Citation: Sumi K (2018) The Japanese Translation of the Life Engagement Test: Reliability and Construct Validity in a College Student Population and a Working Adult Population. Int J Psychol Behav Anal 4: 136. doi: https://doi.org/10.15344/2018/2455-3867/136

Page 5 of 5

21. Sumi K (2008) Optimism, pessimism, social support, and psychological adjustment: A test of a mediation model. J Educ Health Sci 53: 119-125

22. Sumi K (2013) Reliability and validity of Japanese versions of the Flourishing Scale and the Scale of Positive and Negative Experience. Soc Indic Res 118: 601-615.

23. Sumi K (2014) Temporal stability of the Japanese versions of the Flourishing Scale and the Scale of Positive and Negative Experience. $J$ Psychol Psychother 4:140.

24. Nunnally JC Bernstein IH (1994) Psychometric theory (3rd edition). New York: McGraw-Hill, USA, $736 \mathrm{p}$ 